\title{
Minocycline and celecoxib as adjunctive treatments for bipolar depression: a study protocol for a multicenter factorial design randomized controlled trial
}

\author{
This article was published in the following Dove Press journal: \\ Neuropsychiatric Disease and Treatment \\ 19 December 2016 \\ Number of times this article has been viewed
}

\author{
Muhammad I Husain' \\ Imran B Chaudhry ${ }^{2}$ \\ Munir M Hamirani ${ }^{3}$ \\ Fareed A Minhas ${ }^{4}$ \\ Ajmal Kazmi ${ }^{5}$ \\ John Hodsoll ${ }^{6}$ \\ Peter $\mathrm{M} \mathrm{Haddad}^{7}$ \\ John FW Deakin ${ }^{7}$ \\ Nusrat Husain ${ }^{7}$ \\ Allan $\mathrm{H}_{\text {Young }}^{8}$ \\ 'Camden and Islington NHS \\ Foundation Trust, St Pancras \\ Hospital, London, UK; ${ }^{2}$ Pakistan \\ Institute of Learning and Living, \\ ${ }^{3}$ Department of Psychiatry, Abbasi \\ Shaheed Hospital, Karachi, ${ }^{4}$ Institute \\ of Psychiatry, Rawalpindi Medical \\ College, Rawalpindi, ${ }^{5}$ Department \\ of Psychiatry, Karwan-e-Hayat \\ Hospital, Karachi, Pakistan; \\ ${ }^{6}$ Department of Psychological \\ Medicine, Institute of Psychiatry, \\ Psychology and Neuroscience, \\ King's College London, London; \\ ${ }^{7}$ Division of Psychology and Mental \\ Health, University of Manchester, \\ Manchester, ${ }^{8}$ Centre for Affective \\ Disorders, Institute of Psychiatry, \\ Psychology and Neuroscience, \\ King's College London, London, UK
}

Correspondence: Muhammad I Husain Camden and Islington NHS Foundation Trust, St Pancras Hospital, 4 St Pancras Way, London NWI OPE, UK

Tel +4478 62272533

Email ishrat-h@doctors.net.uk
Background: Evidence suggests that the use of anti-inflammatory agents may improve depressive symptoms in patients with bipolar affective disorder. However, there are few welldesigned clinical trials demonstrating the efficacy of these newer treatment strategies.

Patients and methods: This is a multicenter, 3-month, randomized, placebo-controlled, double-blind, factorial design trial of minocycline and/or celecoxib added to TAU for the treatment of depressive symptoms in patients experiencing a DSM-5 bipolar I or II disorder and a current major depressive episode. A total of 240 participants will undergo screening and randomization followed by four assessment visits. The primary outcome measure will be mean change from baseline to week 12 on the Hamilton Depression Scale scores. Clinical assessments using the Clinical Global Impression scale, Patient Health Questionnaire-9, and the Generalized Anxiety Disorder 7-item scale will be carried out at every visit as secondary outcomes. Sideeffect checklists will be used to monitor the adverse events at each visit. Complete blood count and plasma C-reactive protein will be measured at baseline and at the end of the treatment. Minocycline will be started at $100 \mathrm{mg}$ once daily and increased to $200 \mathrm{mg}$ at 2 weeks. Celecoxib will be started at $200 \mathrm{mg}$ once daily and increased to $400 \mathrm{mg}$ at 2 weeks.

Discussion: Anti-inflammatory agents have been shown to be potentially efficacious in the treatment of depressive symptoms. The aim of this study is to determine whether the addition of minocycline and/or celecoxib to TAU improves depressive symptoms in patients with bipolar affective disorder.

Keywords: bipolar disorder, depression, inflammation, anti-inflammatory, minocycline, celecoxib

\section{Background}

Bipolar disorder affects $\sim 2 \%$ of the population and is associated with significant morbidity and mortality. ${ }^{1}$ It is in many cases a chronic and debilitating illness, often with poor recovery between episodes. ${ }^{2,3}$ The current mainstay of treatment is moodstabilizing medication often augmented with antipsychotics. However, there is often a suboptimal response to these treatments with poor tolerance of the drugs due to adverse effects. ${ }^{4-6}$ The depressive phase in both bipolar I and II remains a significant treatment challenge, particularly given the uncertainty surrounding the efficacy and use of antidepressants and the associated risk of manic switch with these drugs. ${ }^{4}$ It has been reported that after initial onset, patients with bipolar disorder have residual submit your manuscript Dovepress f cC. hereby accept the Terms. Non-commercial uses of the work are permitted without any further permission from Dove Medical Press Limited, provided the work is properly attributed. For permission for commercial use of this work, please see paragraphs 4.2 and 5 of our Terms (https://www.dovepress.com/terms.php). 
depressive symptoms for about a third of their lives. ${ }^{7}$ There is a clear need to search for new treatment approaches for these patients.

Inflammatory processes are believed to play a role in psychiatric conditions including depression and bipolar disorder. This hypothesis is based on findings from recent studies in both the preclinical and clinical phases. Multiple reviews have clearly demonstrated that bipolar disorder is associated with abnormal levels of circulating pro- and anti-inflammatory immune biomarkers in the affected patients. ${ }^{8,9}$ Studies have also revealed elevated levels of pro-inflammatory cytokines, TNF- $\alpha$ and IL- 4 , and their receptors sTNFR1 and sIL-2R in patients with bipolar disorder. ${ }^{8}$ However, a recent meta-analysis has indicated that the levels of inflammatory biomarkers in bipolar disorder may be dependent on the mood episode (ie, manic, depressed, and euthymic). ${ }^{10}$ A study in 2015 compared the inflammatory status of patients with bipolar I disorder with healthy controls and found that

[...] those with either mania or depression had significantly elevated levels of TNF- $\alpha$, its soluble receptors (sTNFR1/sTNFR2), other macrophage-derived cytokines (interleukin $1 \beta$ [IL-1 $1 \beta$ ], IL-6, IL-10, and IL-18) in addition to IL-4, interferon- $\gamma$, monocyte chemotactic protein-1, fibroblast growth factor $\beta$ and vascular endothelial growth factor. ${ }^{11}$

The link between inflammatory medical illnesses and higher rates of psychiatric comorbidities (especially major depressive disorder) also strengthens the inflammatory hypothesis for mood disorder. For instance, in patients who suffer from both Crohn's disease and depression, exacerbations of physical illness tend to occur at the same time as depressive episodes. ${ }^{12}$ Furthermore, patients treated with cytokines for various physical health conditions are at an increased risk of developing depression; up to $45 \%$ of patients experience depressive symptoms following treatment with cytokine IFN- $\alpha .{ }^{13,14}$ Bipolar disorder has also been proposed as a multisystemic inflammatory disease due to the significant somatic comorbidity associated with it. ${ }^{15}$

Current evidence suggests that adjunctive antiinflammatory medication may be effective in the treatment of affective disorders. ${ }^{16-19}$ Minocycline is a tetracycline antibiotic that has good penetration through the blood-brain barrier and can affect multiple systems including the antiinflammatory, antioxidant, antiapoptotic, glutamatergic, and monoaminergic pathways. These pathways have all been implicated in the etiology of mood disorders. For this reason, minocycline has previously been proposed for the treatment of depressive symptoms and negative symptoms in schizophrenia. ${ }^{20,21}$ An open-label study of patients with psychotic unipolar depression found that minocycline augmentation of antidepressant treatment was effective in improving depressive symptoms and was well tolerated. ${ }^{22}$ A recent double-blind RCT of minocycline used for mild-tomoderate depression in patients diagnosed with HIV+ found that it was well tolerated and effective in reducing depressive symptoms after 6 weeks of treatment. ${ }^{23}$

Similarly, several clinical trials have shown promising results when investigating celecoxib for the treatment of depressive symptoms. A recent meta-analysis showed that using celecoxib as an add-on to antidepressant therapy led to a significant improvement in symptoms, as well as improving both response and remission in patients with major depressive disorder compared with placebo. ${ }^{19}$ There is currently only one clinical trial that has investigated the use of celecoxib in bipolar depression. ${ }^{24}$ This was an RCT of 28 participants with bipolar disorder experiencing a depressive or mixed episode. The authors found that

patients receiving celecoxib had lower HAM-D scores after one week of treatment compared to the patients receiving placebo, but this difference was not statistically significant,

and by the end of the 6-week trial, there was no difference in depression scores between patients receiving celecoxib and those receiving placebo. ${ }^{24}$

In this factorial design, double-blind RCT, the safety and efficacy of minocycline and/or celecoxib will be determined as adjuncts to TAU in patients experiencing a depressive phase of bipolar I or II disorder. This study hypothesizes that augmentation with minocycline and/or celecoxib will improve depressive symptoms in participants, compared with TAU alone.

\section{Objective}

To investigate whether the addition of minocycline and/or celecoxib to TAU for 12 weeks in patients experiencing a depressive phase of bipolar disorder will lead to an improvement in depressive symptoms compared with TAU.

\section{Patients and methods Study design}

This is a multicenter, 12-week, randomized, double-blind, placebo-controlled, factorial designed trial of minocycline and/or celecoxib added to TAU for patients suffering from DSM-5 bipolar I or II disorder and a current major depressive episode. 
Table I $2 \times 2$ factorial design table showing number of participants in each arm

\begin{tabular}{llll}
\hline & Placebo & Minocycline & Total \\
\hline Placebo & 60 & 60 & 120 \\
Celecoxib & 60 & 60 & 120 \\
Total & 120 & 120 & \\
\hline
\end{tabular}

The study will adopt a $2 \times 2$ factorial design, with 60 completers in each arm, giving a total of 240 participants over the study period (Table 1). The study will be a fourarm trial; one arm will receive minocycline with TAU; the second arm will receive celecoxib with TAU; the third arm will receive both minocycline and celecoxib with TAU; and the fourth arm will receive placebo with TAU.

Recruitment will occur at outpatient psychiatric clinics in Karachi, Lahore, and Rawalpindi, Pakistan. All participants will provide written informed consent after reading the information in English and/or Urdu. TAU will consist of medication including mood stabilizers, antipsychotics, and antidepressants (SSRIs, Tricyclics, MAOIs, and SNRIs), psychotherapy and other psychosocial interventions.

\section{Trial status}

This trial (Clinicaltrials.gov identifier NCT02703363) was registered in March 8, 2016, and is currently recruiting participants. The study completion date is estimated to be August 2018.

\section{Randomization}

A restricted randomization (permuted block randomization) method will be used in which subjects are randomly allocated to each block ( $n=60)$ to ensure that equal numbers of participants receive each drug/placebo combination. An off-site statistician, who will not be involved in clinical assessments, will determine randomization sequences. The statistician will assign the treatment code to the participant after the consenting phase.

Participants, their carers, referring psychiatrists, and the researchers carrying out assessments will be blind to the study drugs until completion. A single pharmacy at each center will be responsible for dispensing minocycline, celecoxib, and placebo in identical tablet form and matched for size, shape, and color. Study medication will be obtained from a local pharmaceutical company that will hold ISO certification.

\section{Sample size}

An effect size of 0.40 or higher is considered clinically significant as criteria for response in clinical trials of antidepressants. ${ }^{25}$ It is also assumed that there is no interaction between the two interventions and thus the study is powered to measure only for the main effects. The sample size is adjusted for $20 \%$ attrition rate due to loss to follow-up and/or dropout. With these assumptions, a sample size of 262 would give $90 \%$ power to detect an SMD of 0.45 at $5 \%$ level of significance.

Sample size was calculated based on a group SMD of $0.45 \mathrm{SD}$ on the HAM-D scores, for either minocycline or celecoxib. This is a moderate size effect and is the minimal that would be of clinical interest.

In addition, it is possible to improve statistical precision by adjusting for baseline HAM-D levels in the primary analysis. If a correlation between baseline and treatment outcome of 0.4 is assumed conservatively, a simple design effect can be calculated according to the formula $1-\rho 2,{ }^{26}$ giving a sample size deflation of 0.84 and a total sample size of 222 .

\section{Ethics approval}

The ethics committee of KMDC in Karachi, Pakistan, has provided IRB approval for the study.

\section{Inclusion criteria}

1. Males or females aged $18-65$ years

2. DSM-5 diagnosis of bipolar I or II disorder and current major depressive disorder

3. Experiencing current depressive symptoms for at least 4 weeks (HAM-D-17 score $\geq 18$ )

4. Willing and able to provide informed consent

5. Taking current medication for at least 4 weeks prior to baseline assessment

6. Able to take medication in tablet form

7. If female and of child-bearing age, agrees to use contraception and consents to monthly pregnancy tests.

\section{Exclusion criteria}

1. Serious physical health condition (eg, HIV, renal, hepatic, cardiac, and serious dermatological disorders such as exfoliative dermatitis and systemic lupus erythematosus)

2. History of allergies/adverse effects to any of the tetracyclines or other anti-inflammatory medication

3. Current treatment with penicillin

4. Current use of anticoagulant medication

5. History of seizures

6. Current use of other antibiotics, other NSAIDs, acetazolamide, or methotrexate

7. Change of psychiatric medications within the preceding 4 weeks

8. History of substance misuse or dependence within the preceding 3 months according to DSM- 5 criteria

9. Pregnant or breast-feeding 
10. Diagnosis of primary psychotic disorder

\section{High risk of suicide}

12. Three or more concurrent manic/hypomanic symptoms.

The criteria for leaving the study are as follows: 1) at the participant's request, 2) at the discretion of the responsible physician or trial investigator (eg, an adverse event and a poor compliance), and 3) pregnancy.

\section{Study procedure}

\section{Recruitment}

Initially, the research team will approach local clinical teams to inform them about the trial and provide them with the inclusion and exclusion criteria. They will then ask psychiatrists in each outpatient department if they are able to identify any patients who may be potentially eligible to take part in the study. The diagnosis of bipolar disorder will be made using DSM-5 criteria, following an unstructured interview led by the treating psychiatrist.

Researchers will work closely with the clinical team to determine whether patients are suitable to participate in the trial. If patients meet the inclusion criteria and the consultant psychiatrist and clinical team agree that they could be potential participants, the consultant will introduce the study to the patient. If the patient then agrees to be contacted by the research team, the research clinician will arrange an appointment to explain the study verbally and provide them with the participant information sheet. The patient will be given at least 24 hours to read and understand this leaflet. After this, if they decide that they are willing to take part, an appointment will be arranged to obtain signed informed consent for trial participation and also signed consent for access to their medical notes.

\section{Screening}

At the first visit, participants will undergo structured diagnostic interviews using the MINI to confirm a diagnosis of DSM-5 bipolar disorder and current major depressive episode. ${ }^{27}$ This tool has been validated for use in the Urdu language and has been used in previous studies in Pakistan. ${ }^{28}$ The HAM-D-17 scale will be used to screen for depression (which must have been present for at least 4 weeks). A HAM-D-17 score $\geq 18$ will be used as the minimum threshold for depression severity. ${ }^{29}$ Other inclusion/exclusion criteria will be checked, consent will be confirmed and pregnancy testing (if appropriate) will be performed.

\section{Follow-up}

Participants will be randomized to take either:

1. Placebo-minocycline plus placebo-celecoxib

2. Active-minocycline plus placebo-celecoxib

3. Placebo-minocycline plus active-celecoxib

4. Active-minocycline plus active-celecoxib.

Participants will continue with the treatment currently provided by their clinical teams. Minocycline will be started at a dose of $100 \mathrm{mg}$ daily and will be increased after 2 weeks to $200 \mathrm{mg}$ daily, which will be taken as a single dose to encourage concordance. Celecoxib will be started at a dose of $200 \mathrm{mg}$ daily and will be increased after 2 weeks to $400 \mathrm{mg}$ daily, which will be taken as a single dose to encourage concordance (Figure 1).

The clinical team and consultant psychiatrist will continue to oversee routine care for each participant although RAs will be contactable for the duration of the study to respond to any concerns. Contacts will be every 2 weeks until study completion (Table 2). A DMC will be constituted to supervise the trial data as they accrue. The DMC will draft a charter to ensure participant safety and will consider stopping the trial if there is an increased frequency of serious adverse events (eg, celecoxib-associated GI bleed or cardiovascular event and minocycline-associated lupus or serum sicknesslike reaction).
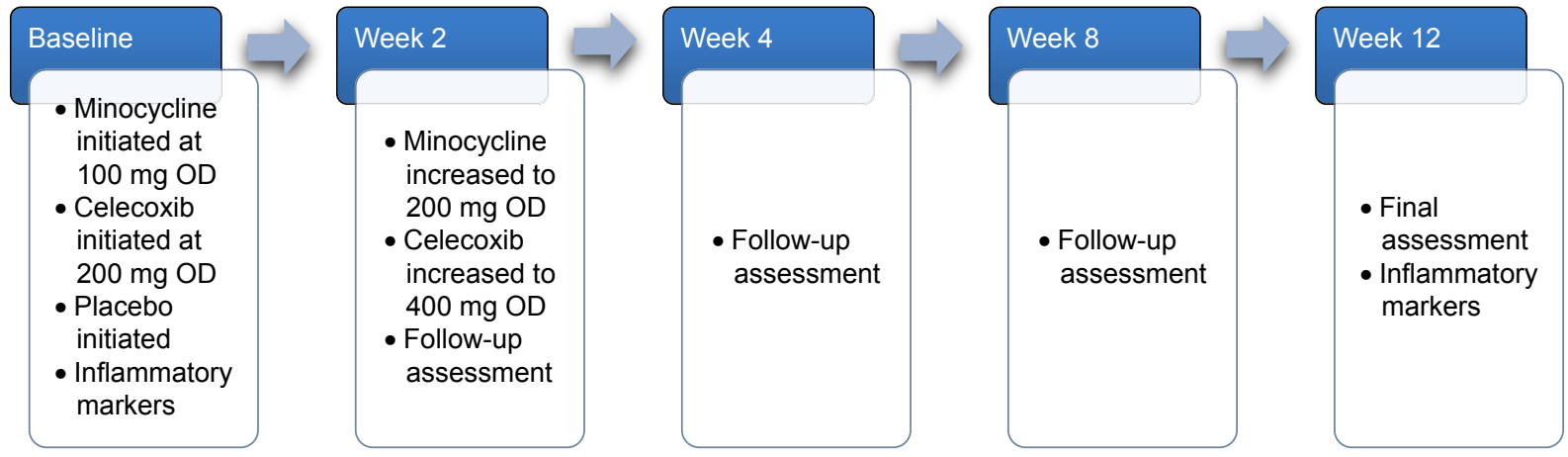

Figure I I2-week assessment schedule. Abbreviation: OD, once daily. 
Table 2 Overall study timeline indicating target milestones

\begin{tabular}{|c|c|c|c|c|c|c|c|c|c|c|c|c|}
\hline Milestones (months) & -3 & $\mathbf{I}$ & 3 & 4 & 5 & 6 & 8 & 12 & 16 & 18 & 22 & 24 \\
\hline \multicolumn{13}{|l|}{ Setting up/ethical approval } \\
\hline \multicolumn{13}{|l|}{ Staff recruitment/training } \\
\hline \multicolumn{13}{|l|}{ Patient recruitment } \\
\hline \multicolumn{13}{|l|}{ Intervention } \\
\hline \multicolumn{13}{|l|}{ Data entry } \\
\hline \multicolumn{13}{|l|}{ Write up and analysis } \\
\hline
\end{tabular}

\section{Outcome measures}

The primary outcome measure will be mean change in HAM-D scale scores from baseline to week 12. For the purposes of this study, response is defined as a reduction of $50 \%$ or more of the HAM-D-17 score and remission is defined as a score of $\leq 7$ of the HAM-D-17. The 24-item HAM-D scale will also be used to measure atypical depressive symptoms. ${ }^{30}$ Ratings will be made on the basis of a semi-structured clinical interview at baseline and at every biweekly follow-up visit until week 12 .

Other outcomes recorded will include the CGI scale, an overall measure of illness severity, the PHQ- $9,{ }^{31}$ a measure of depression severity, and the GAD-7 questionnaire, ${ }^{32}$ a measure of severity for GAD. All scales have been validated for use in the Urdu language and have been used in previous studies in Pakistan. ${ }^{33}$ Adverse effects will be monitored using scales that have been specifically designed for minocycline and celecoxib. One of these rating scales has been used by the authors in a previous study. ${ }^{21}$

\section{Measurement of biomarkers}

Participants will be requested to provide two blood samples (at baseline and at week 12) for analysis. This is optional and does not influence recruitment to the study. Samples will be analyzed to investigate whether there is an association between minocycline/celecoxib and inflammatory markers and whether this relates to the participants' experience of symptom change. The biomarkers analyzed will include complete blood count and CRP.

\section{Inter-rater reliability}

RAs have been trained in SCID and in carrying out clinical assessments by the University of Manchester for a previous grant-funded study. Inter-rater reliability will be measured throughout the study by local investigators. To establish inter-rater reliability, training videos will be used while two raters code them independently and will then be assessed with the intraclass correlation coefficient.

\section{Statistical analysis}

All analyses will be based on the ITT principle with all participants included in the analysis as randomized. The primary outcome at week 12 (and other continuous measures) will be analyzed using linear regression models, adjusted for baseline outcome scores and including study center as fixed effect, with contrasts for minocycline versus non-minocycline and celecoxib versus non-celecoxib. Although the study is not powered to detect an interaction between the two treatment types, a regression model extended to include an interaction term will assess this. If an interaction is detected, the analysis of minocycline and celecoxib alone versus placebo will be presented. ${ }^{34}$ Missing data will be assumed to be missing at random and accounted for with multiple imputation by chained equations (with 100 imputed datasets). ${ }^{35}$ To allow an unbiased estimate of treatment effect, baseline predictors of dropout will be sought and included in the imputation models. Model assumptions (homoscedasticity and normality of distributions) will be checked. Binary outcomes will be 
analyzed using logistic regression and with equivalent design. If enough participants have provided biomarker samples, mediation models will assess potential association of treatment effects on symptom outcomes by inflammatory markers. All analysis will be carried out in R 3.2 and Stata 14.1.

\section{Study coordination}

Local investigators will chair a weekly meeting with RAs to help coordinate the study. The chief investigator will conduct meetings with the research team every 2 weeks via Skype.

\section{Dissemination}

Results will be reported in a peer review journal and will also be communicated at relevant national and international conferences. Study findings will also be shared through local newspapers for a general audience.

\section{Discussion}

Bipolar disorder is a prevalent condition that can have a profound impact on an individual's functioning. The depressive phase in both bipolar I and II subtypes remains a significant treatment challenge and current pharmacological strategies often have poor response, leaving patients with chronic residual symptoms. Recent studies indicate that the use of certain anti-inflammatory agents may lead to an improvement in depressive symptoms.

To our knowledge, there are currently no RCTs that have studied the use of minocycline in bipolar disorder. There is only one RCT investigating the use of celecoxib in bipolar depression, but this study had a small sample size. The factorial design RCT is the first study to look at both minocycline and celecoxib as adjunctive treatments for bipolar depression. The findings from this study may add to current evidence for the treatment of patients with bipolar disorder experiencing depressive symptoms. If this study indicates a trend that minocycline and/or celecoxib are beneficial in treating bipolar depressive symptoms, it will deliver immediate clinical benefit. Both compounds are inexpensive, off-patent drugs that are easily available worldwide. Both could potentially be a cost-effective treatment option in settings with diminishing resources such as low- and middle-income countries as well as other health care services including the NHS in the UK. In the long term, it is believed that the results of this trial will lead to increased treatment options for patients who experience bipolar depression, a condition that is often highly debilitating and challenging to treat.

\section{Abbreviations}

CGI, Clinical Global Impression; CRP, C-reactive protein; DMC, data monitoring committee; DSM-5, Diagnostic and Statistical Manual-5; GAD-7, Generalized Anxiety Disorder-7; GI, gastrointestinal; HAM-D, Hamilton Depression Rating Scale; IFN, interferon; IL, interleukin; IRB, institutional review board; ISO, International Organization of Standardization; ITT, intention to treat; KMDC, Karachi Medical and Dental College; MINI, Mini International Neuropsychiatric Interview; NHS, National Health Service; NIHR, National Institute for Health Research; NSAIDs, nonsteroidal anti-inflammatory drugs; OD, once daily; PHQ-9, Patient Health Questionnaire-9; RA, research assistant; RCT, randomized controlled trial; SCID, Structured Clinical Interview for DSM-IV; SMD, standardized mean difference; SMRI, Stanley Medical Research Institute; TAU, treatment as usual; TNF, tumor necrosis factor.

\section{Acknowledgments}

JH and AHY receive salary support from the NIHR Mental Health Biomedical Research Centre at South London and Maudsley NHS Foundation Trust and King's College London. The authors would like to thank Professor Farhat Jarfri and Mr Ameer Khoso for their contribution to the study design and Professor Haider Naqvi and Doctor Inti Qurashi for their comments on this manuscript. This study has been funded by the SMRI.

\section{Author contributions}

All authors contributed toward data analysis, drafting and critically revising the paper, gave final approval of the version to be published, and agree to be accountable for all aspects of the work.

\section{Disclosure}

IBC, JFWD, and NH have given lectures and advice to Eli Lilly, Bristol Myers Squibb, Lundbeck, Astra Zeneca, and Janssen pharmaceuticals for which they or their employing institution have been reimbursed. MMH has received educational grants and support for academic meetings from Pfizer, Roche, Novartis, and Nabiqasim. AHY has been commissioned to provide lectures and advice to all major pharmaceutical companies with drugs used in affective and related disorders. AHY has undertaken investigator-initiated studies from Astra Zeneca, Eli Lilly, Lundbeck, and Wyeth. PMH has received personal fees for lecturing and/or consultancy 
(including attending advisory boards) from Allergan, Eli Lilly, Galen, Janssen, Lundbeck, Otsuka, Quantum Pharmaceutical, Roche, Servier, Sunovion, Takeda, and Teva. He has received conference support from Janssen, Lundbeck, Otsuka, Servier, and Sunovion. None of the aforementioned companies have a financial interest in this research. The authors report no conflicts of interest in this work.

\section{References}

1. Merikangas KR, Akiskal HS, Angst J, et al. Lifetime and 12-month prevalence of bipolar spectrum disorder in the National Comorbidity Survey replication. Arch Gen Psychiatry. 2007;64(5):543-552.

2. Malhi GS, Ivanovski B, Hadzi-Pavlovic D, Mitchell PB, Vieta E, Sachdev P. Neuropsychological deficits and functional impairment in bipolar depression, hypomania and euthymia. Bipolar Disord. 2007; 9(1-2):114-125.

3. Marotta A, Chiaie RD, Spagna A, et al. Impaired conflict resolution and vigilance in euthymic bipolar disorder. Psychiatry Res. 2015;229(1-2): 490-496.

4. Geddes JR, Miklowitz DJ. Treatment of bipolar disorder. Lancet. 2013; 381(9878):1672-1682

5. Perlis RH, Ostacher MJ, Patel JK, et al. Predictors of recurrence in bipolar disorder: primary outcomes from the Systematic Treatment Enhancement Program for Bipolar Disorder (STEP-BD). Am J Psychiatry. 2006; 163(2):217-224.

6. Vergunst FK, Fekadu A, Wooderson SC, et al. Longitudinal course of symptom severity and fluctuation in patients with treatmentresistant unipolar and bipolar depression. Psychiatry Res. 2013;207(3): 143-149.

7. Judd LL, Akiskal HS, Schettler PJ, et al. The long-term natural history of the weekly symptomatic status of bipolar I disorder. Arch Gen Psychiatry. 2002;59(6):530-537.

8. Baumeister D, Russell A, Pariante CM, Mondelli V. Inflammatory biomarker profiles of mental disorders and their relation to clinical, social and lifestyle factors. Soc Psychiatry Psychiatr Epidemiol. 2014;49(6): 841-849.

9. Goldstein BI, Kemp DE, Soczynska JK, McIntyre RS. Inflammation and the phenomenology, pathophysiology, comorbidity, and treatment of bipolar disorder: a systematic review of the literature. J Clin Psychiatry. 2009;70(8):1078-1090.

10. Munkholm K, Brauner JV, Kessing LV, Vinberg M. Cytokines in bipolar disorder vs. healthy control subjects: a systematic review and meta-analysis. J Psychiatr Res. 2013;47(9):1119-1133.

11. Fiedorowicz JG, Prossin AR, Johnson CP, Christensen GE, Magnotta VA, Wemmie JA. Peripheral inflammation during abnormal mood states in bipolar I disorder. J Affect Disord. 2015;187:172-178.

12. Mardini HE, Kip KE, Wilson JW. Crohn's disease: a two-year prospective study of the association between psychological distress and disease activity. Dig Dis Sci. 2004;49(3):492-497.

13. Van Gool AR, Kruit WH, Engels FK, Stoter G, BanninkM, Eggermont AM. Neuropsychiatric side effects of interferon-alfa therapy. Pharm World Sci. 2003;25(1):11-20.

14. Capuron L, Miller AH. Immune system to brain signaling: neuropsychopharmacological implications. Pharmacol Ther. 2011;130(2): 226-238.

15. Leboyer M, Soreca I, Scott J, et al. Can bipolar disorder be viewed as a multi-system inflammatory disease? J Affect Disord. 2012;141(1):1-10.

16. Ayorech Z, Tracy DK, Baumeister D, Giaroli G. Taking the fuel out of the fire: evidence for the use of anti-inflammatory agents in the treatment of bipolar disorders. J Affect Disord. 2015;174:467-478.
17. Faridhosseini F, Sadeghi R, Farid L, Pourgholami M. Celecoxib: a new augmentation strategy for depressive mood episodes. A systematic review and meta-analysis of randomized placebo-controlled trials. Hum Psychopharmacol. 2014;29(3):216-223.

18. Fond G, Hamdani N, Kapczinski F, et al. Effectiveness and tolerance of anti-inflammatory drugs' add-on therapy in major mental disorders: a systematic qualitative review. Acta Psychiatr Scand. 2014;129(3): 163-179.

19. Kohler O, Benros ME, Nordentoft M, et al. Effect of anti-inflammatory treatment on depression, depressive symptoms, and adverse effects: a systematic review and meta-analysis of randomized clinical trials. JAMA Psychiatry. 2014;71(12):1381-1391.

20. Soczynska JK, Mansur RB, Brietzke E, et al. Novel therapeutic targets in depression: minocycline as a candidate treatment. Behav Brain Res. 2012;235(2):302-317.

21. Chaudhry IB, Hallak J, Husain N, et al. Minocycline benefits negative symptoms in early schizophrenia: a randomised double-blind placebo-controlled clinical trial in patients on standard treatment. J Psychopharmacol. 2012;26(9):1185.

22. Miyaoka T, Wake R, Furuya M, et al. Minocycline as adjunctive therapy for patients with unipolar psychotic depression: an open-label study. Prog Neuropsychopharmacol Biol Psychiatry. 2012;37(2):222-226.

23. Emadi-Kouchak H, Mohammadinejad P, Asadollahi-Amin A, et al. Therapeutic effects of minocycline on mild-to-moderate depression in HIV patients: a double-blind, placebo-controlled, randomized trial. Int Clin Psychopharmacol. 2016;31(1):20-26.

24. Nery FG, Monkul ES, Hatch JP, et al. Celecoxib as an adjunct in the treatment of depressive or mixed episodes of bipolar disorder: a doubleblind, randomized, placebo-controlled study. Hum Psychopharmacol. 2008;23(2):87-94.

25. Faries D, Herrera J, Rayamajhi J, DeBrota D, Demitrack M, Potter WZ. The responsiveness of the Hamilton depression rating scale. JPsychiatr Res. 2000;34(1):3-10.

26. Borm GF, Fransen J, Lemmens WAJG. A simple sample size formula for analysis of covariance in randomized clinical trials. J Clin Epidemiol. 2007;60(12):1234-1238

27. Sheehan DV, Lecrubier Y, Sheehan KH, et al. The Mini-International Neuropsychiatric Interview (M.I.N.I.): the development and validation of a structured diagnostic psychiatric interview for DSM-IV and ICD-10. J Clin Psychiatry. 1998;59(suppl 20):22-33; quiz34-57.

28. Nisar N, Billoo N, Gadit AA. Prevalence of depression and the associated risks factors among adult women in a fishing community. $J$ Pak Med Assoc. 2004;54(10):519-525.

29. Hamilton M. A rating scale for depression. J Neurol Neurosurg Psychiatry. 1960;23:56-62.

30. Hamilton M. Rating depressive patients. J Clin Psychiatry. 1980; 41(12 pt 2):21-24.

31. Kroenke K, Spitzer RL, Williams JB. The PHQ-9: validity of a brief depression severity measure. J Gen Intern Med. 2001;16(9):606-613.

32. Spitzer RL, Kroenke K, Williams JB, Löwe B. A brief measure for assessing generalized anxiety disorder: the GAD-7. Arch Intern Med. 2006;166(10):1092-1097.

33. Husain N, Chaudhry N, Fatima B, et al. Antidepressant and group psychosocial treatment for depression: a rater blind exploratory RCT from a low income country. Behav Cogn Psychother. 2014;42(6): 693-705.

34. Montgomery AA, Peters TJ, Little P. Design, analysis and presentation of factorial randomised controlled trials. BMC Med Res Methodol. 2003;3(1):26.

35. Royston P, White IR. Multiple imputation by chained equations (MICE): implementation in Stata. J Stat Softw. 2011;45(4):1-20. 


\section{Publish your work in this journal}

Neuropsychiatric Disease and Treatment is an international, peerreviewed journal of clinical therapeutics and pharmacology focusing on concise rapid reporting of clinical or pre-clinical studies on a range of neuropsychiatric and neurological disorders. This journal is indexed on PubMed Central, the 'PsycINFO' database and CAS,

and is the official journal of The International Neuropsychiatric Association (INA). The manuscript management system is completely online and includes a very quick and fair peer-review system, which is all easy to use. Visit http://www.dovepress.com/testimonials.php to read real quotes from published authors.

Submit your manuscript here: http://www.dovepress.com/neuropsychiatric-disease-and-treatment-journal 